Щ्)FRANÇAISE

$>\mathrm{DE}$

필 PEAGOGIE

\section{Revue française de pédagogie}

Recherches en éducation

154 | janvier-mars 2006

La construction des politiques d'éducation : de nouveaux rapports entre science et politique

\title{
Maulini Olivier. Questionner pour enseigner et pour apprendre : le rapport au savoir dans la classe
}

postface de Philippe Perrenoud. Issy-les-Moulineaux : ESF, 2005. - 249 p. (Pédagogies)

\section{Bernard Rey}

\section{OpenEdition}

Édition électronique

URL : http://journals.openedition.org/rfp/195

DOI : $10.4000 /$ rfp. 195

ISSN : 2105-2913

Éditeur

ENS Éditions

Édition imprimée

Date de publication : 1 mars 2006

Pagination : 211-213

ISBN : 2-7342-1039

ISSN : 0556-7807

Référence électronique

Bernard Rey, " Maulini Olivier. Questionner pour enseigner et pour apprendre : le rapport au savoir dans la classe », Revue française de pédagogie [En ligne], 154 | janvier-mars 2006, mis en ligne le 14 octobre 2010, consulté le 22 septembre 2020. URL : http://journals.openedition.org/rfp/195 ; DOI : https:// doi.org/10.4000/rfp.195

Ce document a été généré automatiquement le 22 septembre 2020

(c) tous droits réservés 


\section{Maulini Olivier. Questionner pour enseigner et pour apprendre : le rapport au savoir dans la classe}

postface de Philippe Perrenoud. Issy-les-Moulineaux : ESF, 2005. - 249 p. (Pédagogies)

\section{Bernard Rey}

\section{RÉFÉRENCE}

Maulini Olivier. Questionner pour enseigner et pour apprendre : le rapport au savoir dans la classe / postface de Philippe Perrenoud. Issy-les-Moulineaux : ESF, 2005. - 249 p.

(Pédagogies)

1 Que l'école soit un lieu où s'énoncent d'innombrables questions et réponses, chacun le sait. Mais ces questions sont diverses: le maître questionne pour enseigner, l'élève (idéalement) pour apprendre. Comment les questions s'agencent-elles pour assurer non seulement la transmission des savoirs, mais aussi l'institution, chez les élèves, de l'humanité ? Telle est l'entreprise que mène Olivier Maulini, usant généreusement de toutes les ressources possibles. Avant de nous entraîner dans les classes pour observer ce qu'il en est de la pratique questionnante, il nous invite à une réflexion riche et approfondie qui convoque tour à tour les discours pédagogiques et didactiques, la psychologie, la sociologie, la linguistique et la philosophie.

\section{L'étude se déploie en trois temps.}

Dans le premier, Maulini réexamine les grands débats pédagogiques classiques et montre la fonction décisive qu'y occupent les modalités du questionnement scolaire. Dans l'enseignement frontal le maître expose le savoir et quand il pose une question aux élèves, c'est dans un but de vérification. Mais souvent le cours, tout en restant 
magistral, est « dialogué ». L'enseignant pose, aux moments qui lui sont opportuns, des questions aux élèves et choisit dans les réponses celles qui font avancer l'exposé qu'il a prémédité.

Or cette pratique de l'interrogation magistrale a été radicalement critiquée : trop de petites questions étroitement dirigées dans leur intention conduisent à déproblématiser les situations. Ainsi s'établit une double domination du maître, l'une sur le savoir, l'autre sur l'élève. Questionner l'élève empêche qu'il se questionne. D'où la prescription, faite par de nombreux pédagogues, d'inverser les rôles de questionneur et de questionné : c'est à l'enfant de questionner; il faut faire confiance à sa curiosité qu'anime le processus spontané d'adaptation au réel. Si d'aventure les questions tardent, il est loisible à l'adulte de créer une situation qui les suscitera chez l'enfant: c'est tout l'art de la ruse que prônait Rousseau. Ce qui importe, c'est que ce soit l'enfant qui se pose des questions et que le savoir ne vienne que comme réponse à celles-ci.

4 Mais, on le sait, le débat ne s'arrête pas là. Car toute question ne conduit pas à un savoir digne d'intérêt. Ce n'est pas toujours la question spontanée de l'enfant, foisonnante, débridée et versatile, qui ouvre au savoir authentique. On a critiqué les questions du maître au nom du naturel, mais l'école n'est pas naturelle : elle est là précisément pour faire accéder à une culture. Dès lors, sa fonction n'est pas seulement de transmettre des savoirs, mais plus fondamentalement de faire apprendre à poser les bonnes questions. Il y a, à l'école, un apprentissage du questionnement légitime qui conditionne l'apprentissage du savoir.

5 Deux positions s'opposent donc à propos de l'usage des questions, non pas tant d'ailleurs sur les fins que sur les moyens : d'un côté, on dit qu'il faut cesser de poser des questions aux élèves afin qu'ils s'en posent eux-mêmes; de l'autre on rétorque qu'il faut justement leur poser des questions pour qu'ils apprennent à se les poser.

6 Ainsi dans les différentes modalités possibles du questionnement scolaire, Maulini nous invite à retrouver les termes du grand débat autour duquel s'ordonnent depuis des siècles les théories pédagogiques et éducatives. Certes, cette reconstruction a, l'auteur en convient, quelque chose de schématique et d'artificiel. Mais elle a le mérite de faire apparaître les enjeux majeurs de ces décisions minuscules qui, dans le quotidien des classes, commandent la distribution des questions. Car là, le maître joue deux rôles à la fois : d'une part il pose des questions et répond à des questions, mais d'autre part, consciemment ou non, il organise ces échanges. Comment, se demande Maulini, contrôle-t-il l'interaction dont il est dans le même temps participant?

7 Pour répondre à cette question, l'auteur s'oblige à un détour hors l'école, qui constitue le deuxième temps de l'enquête. Qu'est-ce qui se joue, dans les questions des conversations ordinaires, dans ces lieux où la hiérarchie des personnes et des questions ne doit rien à un dispositif institutionnel ?

8 Ce que nous apprend la linguistique pragmatique, c'est que dans le flux de la conversation, une question est toujours plus astreignante vis-à-vis de ce qui suit qu'elle n'est dépendante de ce qui précède. Le questionneur paraît quémander ; mais en fait il dirige, par sa question, l'interaction qui va suivre. Il formule son incertitude, mais par là il circonscrit l'indétermination.

9 Est-ce à dire que, dans l'éducation et la formation, ce sont les questions spontanées «naturelles» de l'enfant qui sont premières? Convoquant alors la psychologie ou, plutôt, les psychologies, l'auteur montre que plutôt que d'opposer nature et culture, il 
convient d'articuler subjectivation et socialisation. Le sujet se constitue hors de luimême, dans la relation à l'autre et le désir (notamment le désir d'apprendre) ne se réduit pas à la pulsion. L'apprentissage ne naît pas d'un questionnement qui viendrait spontanément de l'enfant, ni d'un questionnement qu'imposerait le maitre. Il vient de leur commune interaction.

C'est alors d'un point de vue philosophique que Maulini poursuit la réflexion : peut-on dire, en empruntant la tradition herméneutique, qu'il y a d'abord l'être questionnant et ses questions et que c'est à partir d'elles que s'ébauchent la relation à l'autre et les éventuelles propositions que cette relation permettra de construire? Ou bien faut-il considérer plutôt qu'il y a d'abord, dans la communication instituée, des propositions et que les éventuelles questions naissent du constat d'un désaccord entre ces propositions? C'est à Wittgenstein que l'auteur emprunte la réponse: il n'y a de question possible que par la détermination de ce qui est questionnable et de ce qui ne l'est pas, du discutable et de l'institué. D'où l'on peut tirer ce que Maulini nomme «l'espace logique de l'interrogation » : dans les classes, finalement, l'enseignant a deux possibilités de conduire le questionnement. Soit la question est instituée, mais ce qui peut faire l'objet de discussion, ce sont les propositions qui répondent à cette question. Soit on part d'une proposition qui n'est pas à remettre en cause et ce dont il y a lieu de discuter, ce sont des questions que l'on peut poser à partir d'elle.

11 Vient alors le troisième temps qui est celui de l'observation dans les classes. Il a porté, dans la durée, sur huit classes des deux dernières années de maternelle et des deux premières années du primaire.

12 Même s'il ne s'y attarde guère, il y a un enjeu social à l'entreprise de Maulini : comment faire que les règles du bon questionnement soient acquises par tous les enfants et ne soient pas abandonnées à l'aléatoire de l'origine sociale. Dès lors, on comprend qu'il ait choisi d'étudier ces mécanismes de construction d'un habitus questionnant, au moment de la scolarité où on risque le plus de le voir s'installer, c'est-à-dire à la maternelle et au début du primaire.

13 En relatant des moments de classe et des passages d'entretiens avec les enseignants, l'auteur décrit successivement comment ceux-ci sélectionnent les questions parmi celles qui viennent spontanément des enfants, puis comment ils produisent eux-mêmes de l'interrogation en attribuant des questions aux élèves ou en proposant leurs propres questions. Enfin l'auteur tente de saisir selon quels principes, conscients ou inconscients, les maîtres opèrent le réglage du questionnement dans la classe.

14 Cette troisième étape, très riche, intéressera au plus haut point les praticiens et les formateurs, mais aussi les chercheurs dont l'objet est la pratique enseignante. Les moments d'interactions entre élèves et maîtres sont très suggestifs et font apparaître, d'une manière convaincante, l'importance décisive du questionnement dans la dramaturgie didactique. On regrette presque de ne pas s'en voir offrir un plus grand nombre. On regrette aussi que, pour chaque moment de classe rapporté, on ne nous indique pas le degré auquel la classe appartient (moyenne ou grande section de maternelle, $\mathrm{CP}$ ou $\mathrm{CE} 1$ ou encore classe à plusieurs niveaux). Car les enfants de huit ans sont très différents de ceux de quatre ans sur beaucoup de points et, notamment, sur ce qui intéresse l'auteur, c'est-à-dire leur plus ou moins grande incorporation du questionnement scolairement légitime.

On est surpris enfin que l'auteur n'utilise pas, comme moyen d'analyse de ce qu'il observe dans les classes, le modèle qu'il présente dans sa deuxième partie sous le nom 
« d'espace logique de l'interrogation ». Mais s'il ne le fait pas, c'est, nous dit-il, avec le souci de ne pas s'ériger en juge des pratiques enseignantes; celles-ci en retour lui permettent, dans les dernières pages, d'enrichir et de nuancer le modèle.

Il faut encore avertir le futur lecteur de cet ouvrage qu'il n'est pas toujours d'un abord facile. Olivier Maulini écrit en phrases courtes qui prétendent en dire long. Elles prennent même, ça et là, l'allure d'aphorismes et cultivent la métaphore. Est-ce le signe d'une impuissance de l'auteur à porter sa pensée dans la pleine clarté ? N'est-ce pas plutôt qu'il est passé maître dans la stratégie de conduire le lecteur à se poser des questions?

Quoi qu'il en soit, le lecteur sera récompensé de ses efforts. Cet ouvrage réalise ce que tous les livres de pédagogie devraient faire : allier la réflexion la plus profonde et la plus savante à l'observation humble et patiente de la vie des classes.

\section{AUTEURS}

BERNARD REY

Université libre de Bruxelles 